\title{
Suppression of KLF8 induces cell differentiation and sensitizes colorectal cancer to 5-fluorouracil
}

\author{
XINPENG SHI $^{1 *}$, XIAOYONG LUO ${ }^{2 *}$, QINGQING YAN ${ }^{1}$, WENJING ZHANG $^{1}$, YAO WU ${ }^{1}$, MENGNAN ZHANG $^{3}$, \\ JINJUN ZHAO ${ }^{1}$, YING PENG ${ }^{1}$, YE CHEN $^{1}$, YALI ZHANG ${ }^{1}, \mathrm{CUNLONG} \mathrm{CHEN}^{1}$, TIANMING CHENG ${ }^{1}$, \\ CHUDI CHEN $^{1}$, SIDE LIU ${ }^{1}$, YANG BAI ${ }^{1}$ and JIDE WANG ${ }^{1}$ \\ ${ }^{1}$ Guangdong Provincial Key Laboratory of Gastroenterology, Department of Gastroenterology, Nanfang Hospital, \\ Southern Medical University, Guangzhou, Guangdong; ${ }^{2}$ Department of Oncology, The Affiliated Luoyang Central Hospital of \\ Zhengzhou University, Luoyang, Henan; ${ }^{3}$ Department of Gastroenterology, The First Affiliated Hospital \\ of Guangzhou Medical University, Guangzhou, Guangdong, P.R. China
}

Received April 8, 2015; Accepted May 20, 2015

DOI: 10.3892/or.2015.4094

\begin{abstract}
KLF8 is a member of the KLF transcription factor family that plays an important role in oncogenesis. However, the role of KLF8 in colorectal cancer remains unknown. The aims of the present study were to examine KLF8 expression in colorectal cancers, to determine the role of KLF8 in cell differentiation and to investigate the antiproliferative effect of KLF8 silencing. The expression of KLF8 and phospho-ERK proteins was analyzed, and the effects of KLF8 suppression on cell differentiation and growth were evaluated. In addition, the biological impact of KLF8 knockdown on colorectal cancer cells was investigated in vitro and in vivo. The expression of the KLF8 protein was higher in 10/14 (71.43\%) fresh cancer tissues compared with adjacent normal tissues, and the blockade of ERK signaling by U0126 decreased the expression of KLF8 in a time- and dose-dependent manner. Furthermore, KLF8-siRNA induced the expression of carcinoembryonic antigen (CEA) and E-cadherin as well as the maturation of F-actin. KLF8 suppression inhibited serum-dependent, anchorage-dependent and -independent cell growth. Moreover, KLF8 silencing
\end{abstract}

Correspondence to: Professor Jide Wang or Professor Yang Bai, Department of Gastroenterology, Nanfang Hospital, Southern Medical University, 1838 North Guangzhou Avenue, Guangzhou, Guangdong 510515, P.R. China

E-mail: jidewang55@163.com

E-mail: baiyang1030@hotmail.com

${ }^{*}$ Contributed equally

Abbreviations: KLF, Krüppel-like factors; EMT, epithelial-tomesenchymal transition; NaB, sodium butyrate; HEK293, human embryonic kidney 293; ATCC, American Type Culture Collection; FBS, fetal bovine serum; src siRNA, scrambled siRNA; ERK, extracellular signal-regulated protein kinases

Key words: KLF8, ERK1/2, F-actin, siRNA, lentivirus, colorectal cancer induced apoptosis and sensitized cancer cells to 5-fluorouracil (5-FU). A strong antitumorigenic effect by lenti-KLF8-shRNA, which was enhanced when combined with 5-FU treatment, was exerted in nude mice. Thus, KLF8 suppression induced cell differentiation and inhibited tumorigenesis.

\section{Introduction}

Colorectal neoplasia is one of the most common malignancies in the Western world. Despite evidence that the 5-year survival from colorectal cancer is $90 \%$ when diagnosed at an early stage, $<40 \%$ of cases are diagnosed when the cancer is localized $(1,2)$. Although the specific underlying mechanisms of action in cancer progression are just beginning to be unraveled, it is believed that colorectal cancer results from a combination of genetic and environmental factors, including the expression of several inherited susceptibility genes for colorectal cancer (3), ethnicity and family history (4), and different dietary (5), and lifestyle factors (6). The reported molecular and biochemical mechanisms that may contribute to the phenotypic changes that favor carcinogenesis, and to the development and progression of the cancer include inhibited apoptosis, enhanced tumor cell proliferation (7), increased invasiveness, perturbed cell adhesion (8), promotion of angiogenesis (9) and inhibited immune surveillance (10).

Krüppel-like factors (KLFs) belong to a class of zinc finger-containing transcription factors that share homology with the general transcription factor Sp1 (11). The members of this family, which contains over 26 identified members, are characterized by a highly conserved C-terminus containing three zinc finger DNA-binding domains (12). Emerging evidence has suggested that KLFs may be critical factors for tumor development, growth and metastasis, and several KLF members, including KLF4 (13), KLF5 (14) and KLF11 (15), have been reported to be associated with the oncogenesis of various types of human cancer. KLF8 binds to GC boxes at a number of gene promoters and regulates their transcription. Previous findings have demonstrated that KLF8 is highly expressed in several types of human cancer, including breast (16) and renal carcinomas (17), and that this protein plays an important role 
in oncogenic transformation. However, the role of KLF8 in the proliferation, differentiation and apoptosis of colorectal cells remains elusive.

In the present study, we first showed that KLF8 was highly expressed in colorectal cancer tissues and cell lines. We then demonstrated that KLF8 repression significantly induced differentiation and apoptosis, inhibited tumorigenesis and tumor growth, and strongly enhanced the antitumor activity of 5-fluorouracil (FU). In vivo, lentivirus-mediated shRNA knockdown of KLF8 suppressed the growth of tumor xenografts in nude mice. Therefore, KLF8 knockdown is considered a potential therapeutic target for treating colorectal cancer.

\section{Materials and methods}

Chemicals, tissue specimens and cell lines. Sodium butyrate (NaB), 5-fluorouracil (5-FU) and U-0126 (a selective MAP kinase inhibitor) were purchased from Sigma (St. Louis, MO, USA), and Rhodamine-phallotoxin was purchased from Molecular Probes (Eugene, OR, USA). Goat anti-human KLF8 and GAPDH antibodies were purchased from Aviva Systems Biology (San Diego, CA, USA). For western blot analyses, 14 pairs of colorectal cancer and adjacent normal tissues $(>5 \mathrm{~cm}$ from the margin of the tumor) were obtained from patients by surgical resection in the Nanfang Hospital (Guangzhou, China). Informed consent was obtained from all individual participants included in the present study. The Medical Ethics Committee of Nanfang Hospital approved the use of tissue specimens in the present study (permit no. NFYY-2013-27). The tissue specimens were snap-frozen in liquid N2 and stored at $-70^{\circ} \mathrm{C}$ until use. The tissues were embedded in paraffin, sliced and then subjected to histopathologic review using immunohistochemistry (8). Tissues in which $>10 \%$ of cancer cells were positively stained were considered positive.

The HCT116, HT29, LoVo, SW620 and SW480 colorectal cancer cell lines and the human embryonic kidney 293 (HEK293) cell line were obtained from ATCC (Rockville, MD, USA) and cultured in RPMI-1640 medium as previously described (7).

RNA isolation and reverse transcription-PCR analysis. Total RNA was isolated using TRI reagent (Sigma) (7) and then reversed transcribed using SuperScript II (Invitrogen-Life Technologies, Carlsbad, CA, USA) according to the manufacturer's instructions. The primer sequences used were: carcinoembryonic antigen (CEA) forward, 5'-AACCCTTCAT CACCAGCAAC-3' and reverse, 5'-CAGGAGAGGCTGAGG TTCAC-3'; E-cadherin forward, 5'-TGCCCAGAAAATGAA AAAGG-3' and E-reverse, 5'-GTGTATGTGGCAATGCG TTC-3'; and GAPDH forward, 5'-GTCAACGGATTTGGT CGTATTG-3' and reverse, 5'-CTCCTGGAAGATGGTGAT GGG-3'. The expected sizes of the PCR products were 340, 200 and 204 bp for CEA, E-cadherin and GAPDH, respectively.

Western blot analysis. Total protein lysates were prepared and submitted to western blotting. The blots were probed with primary antibody followed by horseradish peroxidase-conjugated secondary antibody. Antigen-antibody complexes were visualized using the enhanced chemiluminescence system (Amersham Biosciences, Little Chalfont, UK).
Assay of anchorage-independent and-dependent cell growth. Scrambled siRNA (src siRNA) and KLF8-siRNA-transfected cells were plated in triplicate in plates containing $0.35 \%$ agar on top of a $0.7 \%$ agar base (7). Colonies were scored using Coomassie blue staining, and only colonies containing $\geq 50$ cells were considered viable. The cell proliferation reagent WST-1, a ready-to-use colorimetric assay (Roche Diagnostics), was also used.

Apoptosis assay. Apoptosis was detected in cells using the Annexin V-FITC kit according to the manufacturer's instructions (18) (Trevigen, Inc., Gaithersburg, MD, USA) followed by flow cytometry using WinMDI 2.9. Apoptosis was also analyzed by staining cell nuclei with Hoechst 33258 and examining the cells under a fluorescence microscope. The activities of caspases 3,8 and 9 were determined using the ApoAlert caspase colorimetric assay kit according to the manufacturer's instructions (18) (Clontech, Mountain View, CA, USA).

Immunofluorescence. For F-actin staining (7), the cells were fixed on coverslips and incubated with Rhodamine-conjugated phallotoxin (5 U/ml; Molecular Probes) in phosphate-buffered saline (PBS) at room temperature. In addition, the nuclei were stained with $1 \mu \mathrm{g} / \mathrm{ml}$ Hoechst 33258. The coverslips were then washed, mounted and visualized using a Zeiss Axioskop fluorescence microscope.

siRNA transfection in vitro. The siRNA duplexes consisted of 21 base pairs with a 2-base deoxynucleotide overhang (Proligo, Singapore). The sequence of the KLF8-siRNA: (NM_007250 sense strand, CGAUAUGGAUAAACUCAUATT) was identical to that employed in a previous study (19), and src siRNA, (5'-TTCTCCGAACGTGTCACGT-3'), which did not target any genes, was used as the negative control. The cells were transfected with siRNA duplexes using oligofectamine (Invitrogen-Life Technologies) according to the manufacturer's instructions.

Construction and transfection of lentiviral vectors containing $K L F 8$ short hairpin RNA. To investigate the effects of siRNAinduced downregulation of KLF8 expression on in vivo tumor growth,aKLF8-RNAilentiviralvector(pGCSIL-KLF8shRNA) was constructed (Shanghai GeneChem Co., Ltd., Shanghai, China) (20). A GFP-lentiviral vector (pGCSIL-GFP) was used as a negative control. Double-stranded oligonucleotides encoding human KLF8-vshRNA (CCGGCTAGCATGCTA CAAGCTCCA-ATTCAAGAGATTGGAGCTTGTAGCA TGCTAGTTTTTG) were inserted into the short hairpin RNA (shRNA) expression vector pGCSIL (Shanghai GeneChem Co., Ltd.), and the identities of the clone were verified by sequencing.

A recombinant lentiviral vector was produced by co-transfecting HEK293T cells with the lentiviral expression vector and the packaging plasmid mix using Lipofectamine ${ }^{\mathrm{TM}} 2000$ according to the manufacturer's instructions (19). Infectious lentiviral particles were harvested at $48 \mathrm{~h}$ post-transfection and then filtered through $0.45-\mu \mathrm{m}$ cellulose acetate filters. The virus was concentrated, and the titer was determined by serial dilution on 293T cells. 

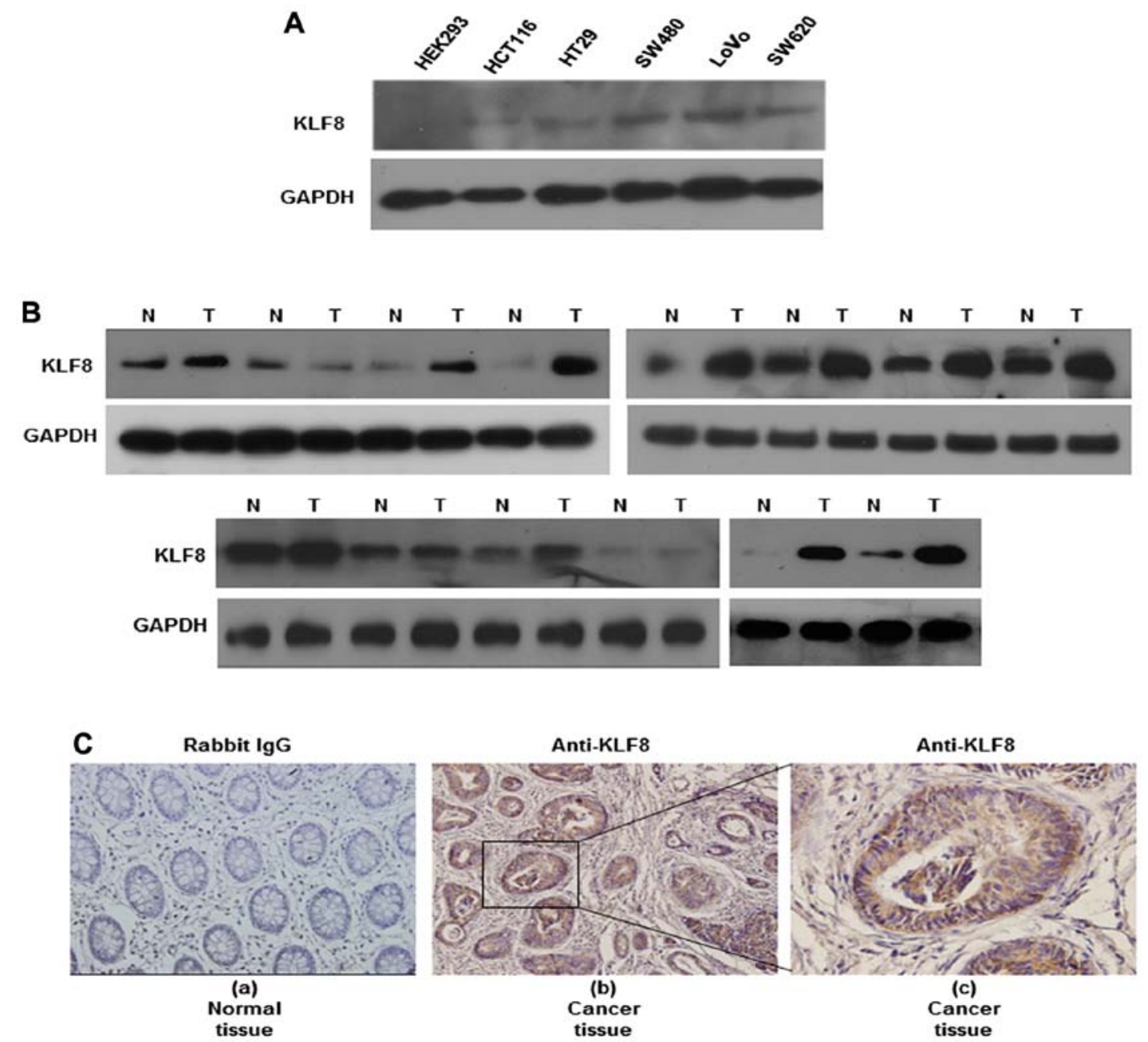

Figure 1. Colorectal cancer tissues express higher levels of KLF8 than normal tissues. (A) Whole lysates of HEK293, HCT116, HT29, SW480, LoVo and SW620 cells were collected and KLF8 expression was detected by western blotting. (B) Proteins isolated from resected tumor and adjacent non-tumorous tissue specimens were subjected to western blot analysis. T, colorectal cancer tissues; N, normal tissues. GAPDH was used as the internal control (GAPDH, glyceraldehyde-3-phosphate dehydrogenase). (C) KLF8 expression in normal (a) or cancerous colon tissue specimens (b and c) was detected by immunohistochemistry assays. Original magnification, x200. The experiments were repeated three times with identical findings.

For the lentiviral transduction, LoVo cells were subcultured at $1 \times 10^{5}$ cells/well in 6-well culture plates and then transduced with KLF8-siRNA-expressing (KLF8-siRNA) or src siRNA-expressing lentivirus at a multiplicity of infection (MOI) of 50. The cells were collected $72 \mathrm{~h}$ after infection, and the transduction efficiency was evaluated by counting the percentage of GFP-positive cells.

Xenograft tumor model. The present study was carried out strictly in accordance with the recommendations in the Guide for the IACUC (Institutional Animal Care and Use Committee), and the protocol was approved by the Committee on the Ethics of Animal Experiments of Nanfang Hospital (permit no. NFYY-2013-36). Surgeries were performed under sodium pentobarbital anesthesia, and all efforts were made to minimize suffering. After the surgery, the nude mice were euthanized by sodium pentobarbital anesthesia.

The antitumor effects due to transfection of the KLF8siRNA were evaluated in vivo using nude mice xenograft models (21). Five- to six-week-old female BALB/c nude mice were bred under pathogen-free conditions at the Southern
Medical University (Guangzhou, China), and all the animal studies were approved by the Southern Medical University Animal Care and Use Committee. Lenti-src-shRNA- and lenti-KLF8-shRNA-infected LoVo cells were harvested during the exponential growth phase, washed twice in PBS and resuspended in PBS at a density of $5 \times 10^{7}$ cells $/ \mathrm{ml}$. The cell suspension $\left(0.1 \mathrm{ml} ; 5 \times 10^{6}\right.$ cells $)$ was then subcutaneously injected into the right flank of each nude mouse. LoVo cells infected with lenti-src-shRNA were injected into groups a and c, respectively. Lenti-KLF8-shRNA-infected LoVo cells were injected into group $b$ and d. Fourteen days after the subcutaneous LoVo cell injection, 5-FU was injected into mouse groups $\mathrm{c}$ and $\mathrm{d}$ through intraperitoneal injection using a 10-ml micro-syringe (Hamilton, Reno, NV, USA), with the dose of $50 \mu \mathrm{g} / \mathrm{kg} / \mathrm{time}$, once/two days, 12 times. Five mice were included in each group (for each transduced cell line), and the tumor volumes were calculated as: $V=(4 / 3) R 1^{2} R 2$, where $\mathrm{R} 1$ is radius $1, \mathrm{R} 2$ is radius 2 and $\mathrm{R} 1<\mathrm{R} 2$. The mice were then sacrificed, and the tumors were dissected, snap-frozen in liquid $\mathrm{N} 2$ and stored at $-70^{\circ} \mathrm{C}$ on day 42 after inoculation for western blotting. 


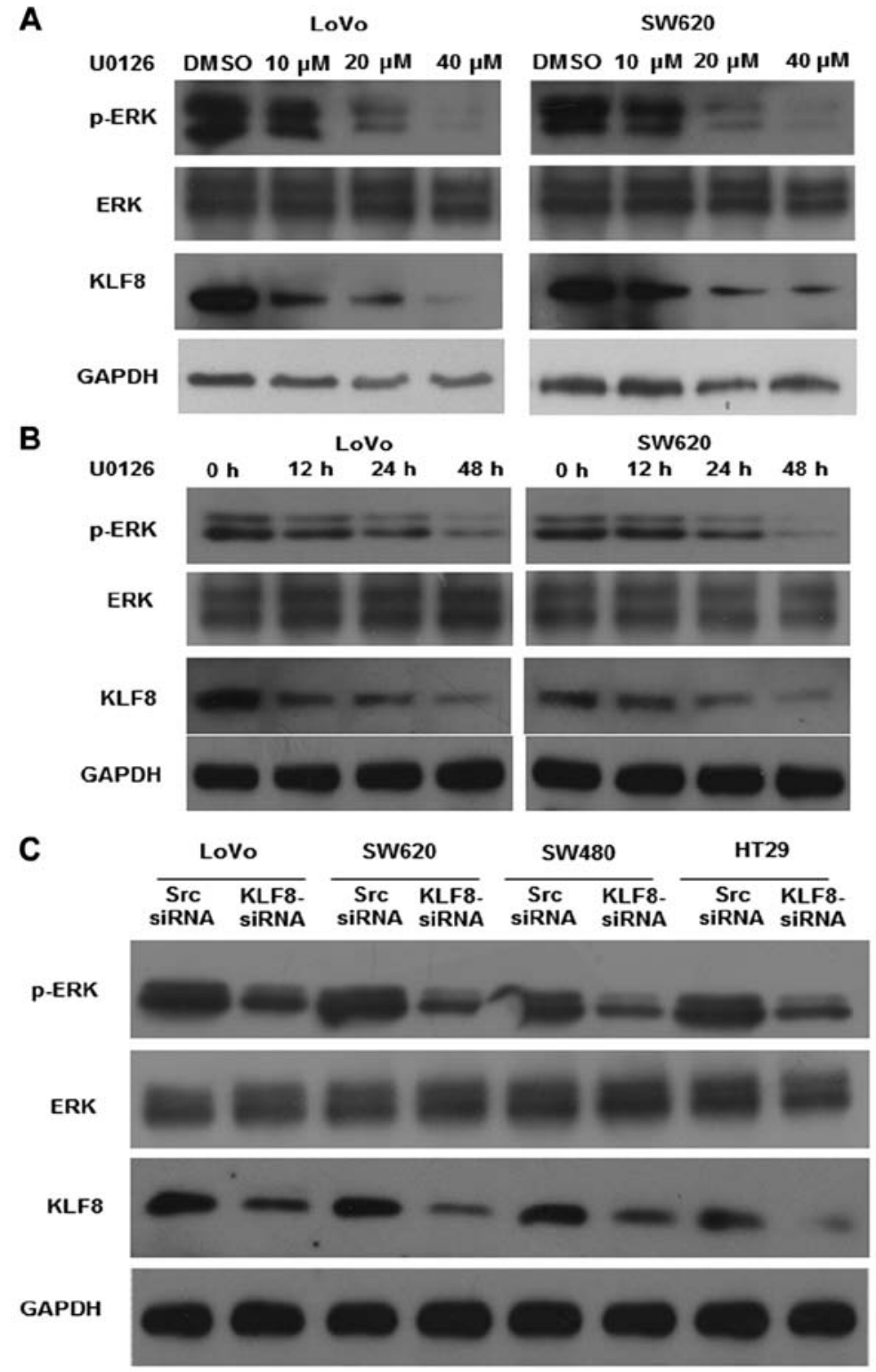

Figure 2. Inhibition of ERK1/2 downregulates KLF8 expression. (A) LoVo and SW620 cells were treated with the indicated concentrations of U0126 for $48 \mathrm{~h}$. (B) LoVo and SW620 cells were treated with $20 \mu \mathrm{M}$ U0126 for the indicated times. Expression of ERK1/2, p-ERK1/2 and KLF8 was detected by western blot analysis. GAPDH was used as the internal control. DMSO, dimethylsulfoxide. (C) Cells were transfected with KLF8-siRNA or src-siRNA (scramble siRNA) for $48 \mathrm{~h}$, followed by western blot analysis. The experiments were repeated three times with similar findings.

Statistical analysis. Results obtained from in vitro and in vivo experiments were presented as means \pm SD and statistically evaluated using the standard two-tailed Student's t-test or one-way ANOVA. If equal variances were not assumed, the Satterthwaite's t-test or Dunnett's T3 test were used to analyze the quantified data. The results were considered significant when $\mathrm{P}<0.05$.

\section{Results}

Colon cancer tissues express higher levels of KLF8 protein than normal tissues. We first showed that KLF8 protein expression was significantly increased in the HCT116, HT29, LoVo, SW620 and SW480 cancer cell lines, compared with that in the human HEK293 epithelial cell line. We then measured KLF8 expression in matched normal $(\mathrm{N})$ and cancerous $(\mathrm{T})$ colon tissues by western blotting. Of the 14 cancerous tissues,
10 expressed higher levels of KLF8 than the normal tissues (Fig. 1B). KLF8 expression in tissue specimens collected from non-cancerous or cancerous colons was also measured in situ using immunohistochemistry. We found nuclear-specific KLF8 protein expression in the carcinoma cells of all the colorectal cancer samples. However, KLF8 was not expressed in the control tissue, as exemplified in Fig. 1C (b and c). Fig. 1C shows representative images of KLF8 expression in non-cancerous and cancerous specimens. These findings demonstrated that KLF8 was overexpressed in colorectal cancer.

Inhibition of the constitutive activation of ERK1/2 downregulates KLF8 expression. Previous studies have indicated that activation of the MAPK/ERK pathway is involved in the regulation of cell proliferation in colorectal cancer $(2,21)$. Therefore, we analyzed the effect of ERK inhibition on KLF8 expression after incubating LoVo and SW620 cells with U0126, a specific 
A
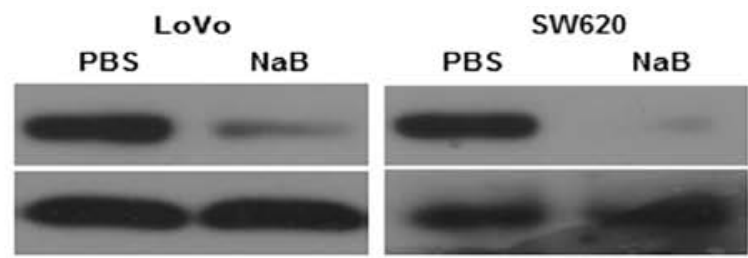

B

C
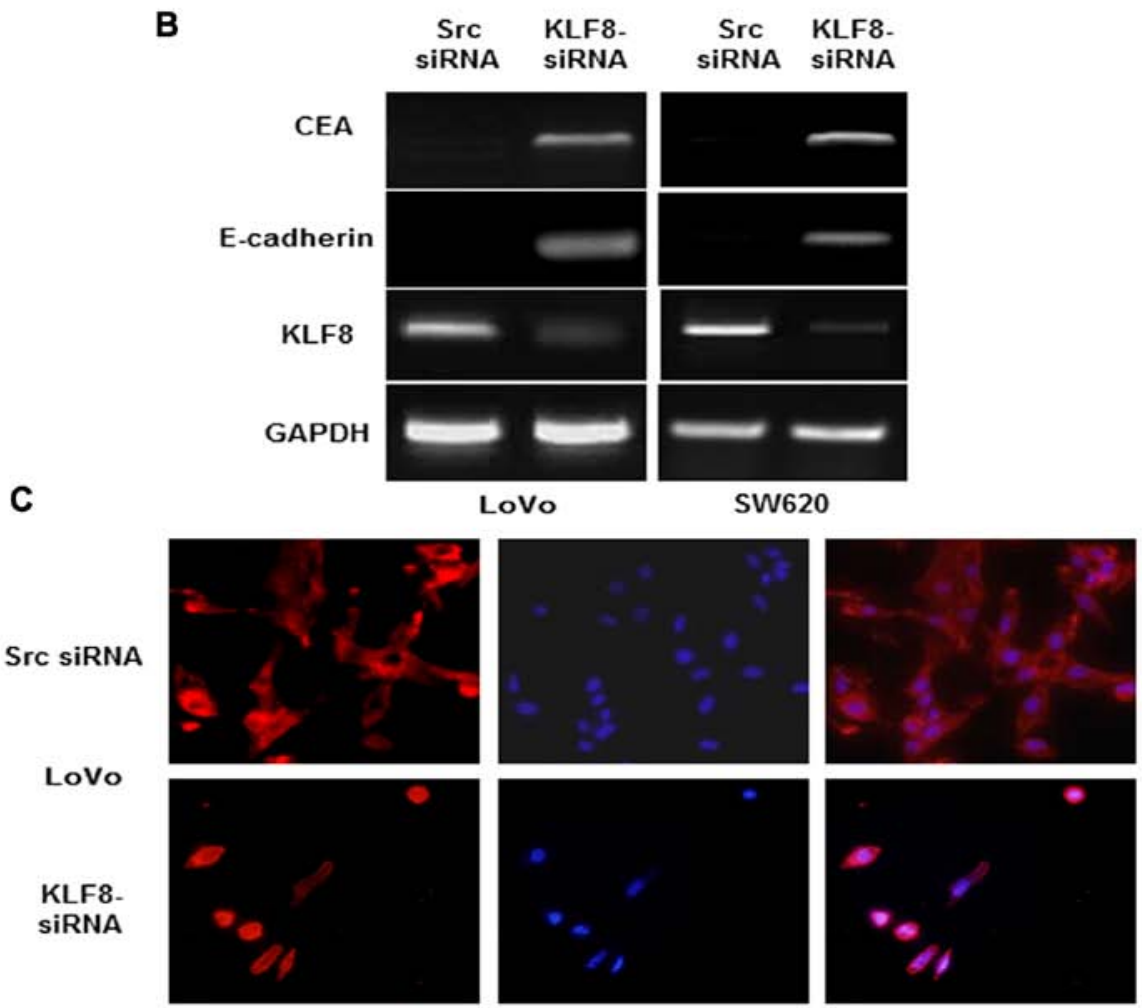



Rhodaminephalloidin
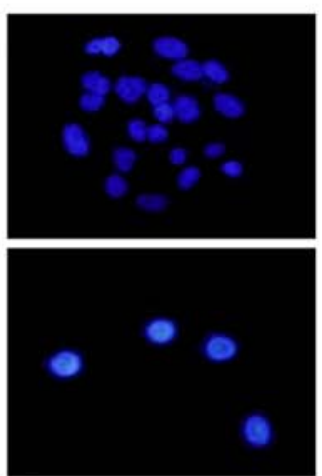

Hoechst 33258
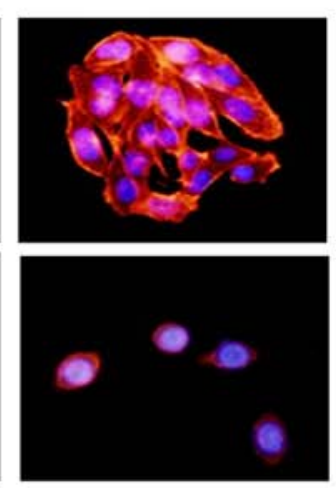

Merge

Figure 3. Suppression of KLF8 with siRNA induces cell differentiation in cancer cells. (A) KLF8 expression in LoVo and SW620 cells treated with or without sodium butyrate $(\mathrm{NaB})$ for $48 \mathrm{~h}$, as detected by western blotting. (B) Expression of CEA and E-cadherin in cells transfected with KLF8-siRNA or src siRNA for $48 \mathrm{~h}$, as detected by RT-PCR. (C) LoVo and SW620 cells transfected with KLF8-siRNA or src siRNA were stained with Rhodamine-phallotoxin for $48 \mathrm{~h}$, and F-actin filaments were visualized using fluorescence microscopy. These images are representative of three independent experiments with identical findings. CEA, carcinoembryonic antigen.

MEK/ERK activation inhibitor, for $48 \mathrm{~h}$. The results showed that the expression of pERK1/2 and KLF8 was significantly downregulated in a dose- and time-dependent manner after U0126 treatment (Fig. 2A and B).

By contrast, KLF8 knockdown affected the activation of ERK. After confirming the efficiency of siRNA knockdown by western blotting (Fig. 2C), the expression of pERK1/2 was found to be significantly reduced after KLF8 knockdown.
However, no change in the expression of ERK1/2 was observed. These findings suggested that KLF8 inhibition is important in ERK activation in colorectal cancer.

Suppression of KLF8-induced cell differentiation in cancer cells. To examine the effect of KLF8 on cell differentiation, we first investigated the effects of pro-differentiation agents on KLF8 expression (22). LoVo and SW620 cells were treated 

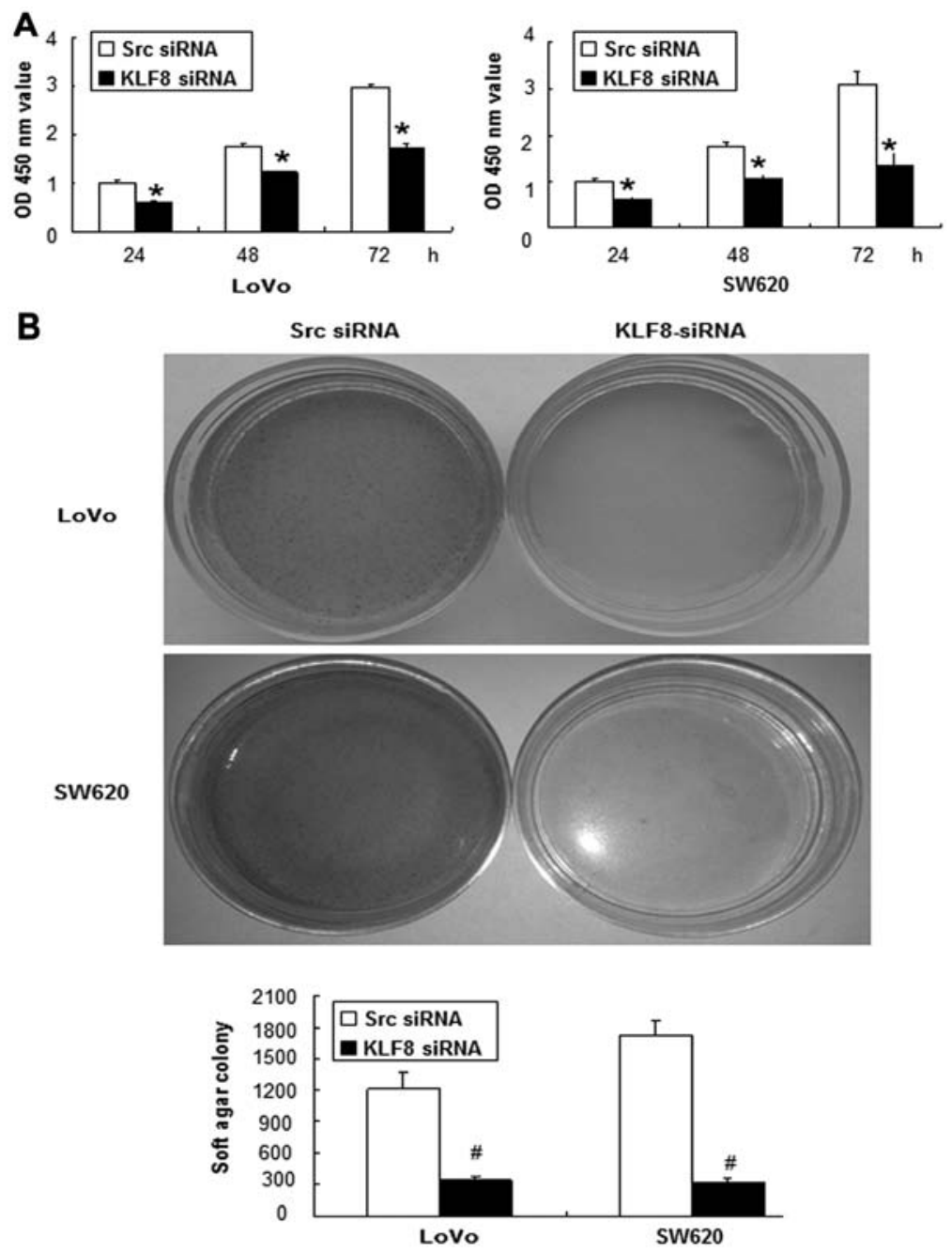

Figure 4. KLF8 repression inhibits anchorage-dependent and anchorage-independent cell growth. (A) LoVo and SW620 cells were transfected with KLF8-siRNA or src siRNA, and a WST-1 assay was performed; " $\mathrm{p}<0.05$. (B) KLF8-repressed LoVo and SW620 cells and their corresponding control cells $\left(5 \times 10^{3}\right)$ were plated in tissue culture dishes with complete culture medium containing $0.35 \%$ agar on the top and $0.5 \%$ agar on the bottom. After 14 days, cell colonies were visualized by staining with $0.005 \%$ crystal violet. Colonies containing $>50$ cells were considered viable, and the results are presented as the means $\pm \mathrm{SD} . "{ }^{*} \mathrm{p}<0.05$. The experiments were repeated three times with similar findings.

with $\mathrm{NaB}$, which significantly reduced the expression of KLF8 (Fig. 3A). Using RT-PCR we showed that, KLF8 knockdown markedly increased the expression of the differentiation markers CEA and E-cadherin (7,23) (Fig. 3B). In addition, cell morphology was observed using phalloidin staining to detect F-actin localization $(7,24)$. Diffuse and generally uniform distribution throughout the cytoplasm of F-actin transfected with KLF8-siRNA was exhibited. However, src siRNA transfected into a human colon cancer cell line presented by multiple clumps of apparently aggregate size at the rim zone of the protrusion, requiring actin polymerization (Fig. 3C). Thus, the above findings suggested that knockdown of KLF8 induced differentiation of colorectal cancer cells.

Knockdown of KLF8 inhibits anchorage-dependent and anchorage-independent growth. We assessed the effect of KLF8 repression on the growth characteristics of colorectal cancer cells using the WST-1 assay (25). We showed that the growth rates of cells transfected with the LoVo-KLF8-siRNA after culture for 24,48 and $72 \mathrm{~h}$, were $0.6 \pm 0.05,1.22 \pm 0.01$ and $1.72 \pm 0.09 \%$, respectively, whereas those of LoVo-src siRNA cells were $1 \pm 0.05,1.74 \pm 0.07$ and $2.97 \pm 0.05 \%$, respectively (Fig. 4A). Significant differences between the growth rates of LoVo-KLF8-siRNA- and LoVo-src siRNA-transfected cells were found at all three time points $(\mathrm{p}<0.05)$, and similar results were observed for SW620 cells (Fig. 4A).

Anchorage-independent growth is a pivotal characteristic of malignant transformation (26). Therefore, a soft-agar assay was carried out to identify the function of KLF8. As expected, KLF8 repression for 14 days significantly inhibited the colony-forming ability of LoVo and SW620 cells (Fig. 4B).

These results showed that KLF8 knockdown inhibited anchorage-dependent and -independent growth of colorectal cancer cells.

Repression of KLF8 induces apoptosis and sensitizes cancer cells to chemotherapeutic 5-FU. To investigate the mechanism by which KLF8 knockdown induces growth suppression, an apoptosis assay was performed using Annexin V-FITC and PI double staining followed by flow cytometry. As shown in Fig. 5A, the percentage of apoptotic cells was significantly increased from $0.81 \%$ in src siRNA-transfected LoVo cells to 
A

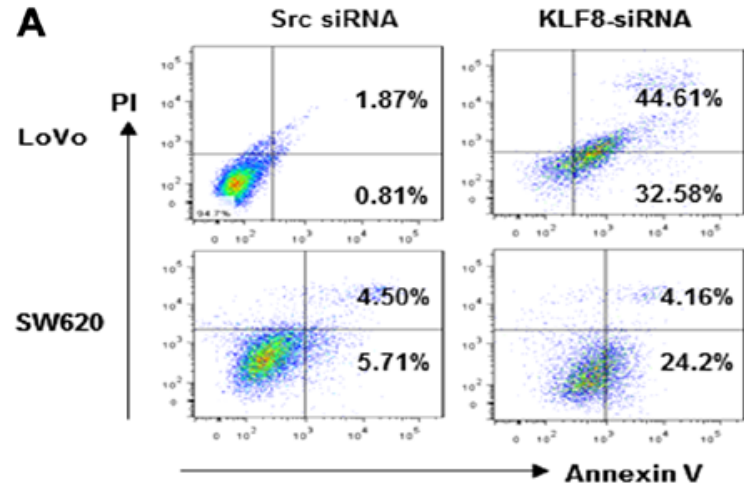

C

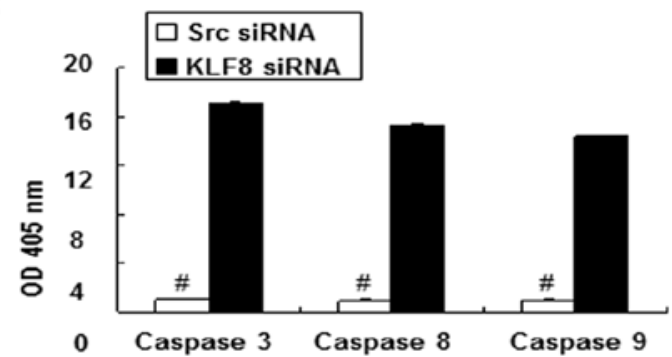

D

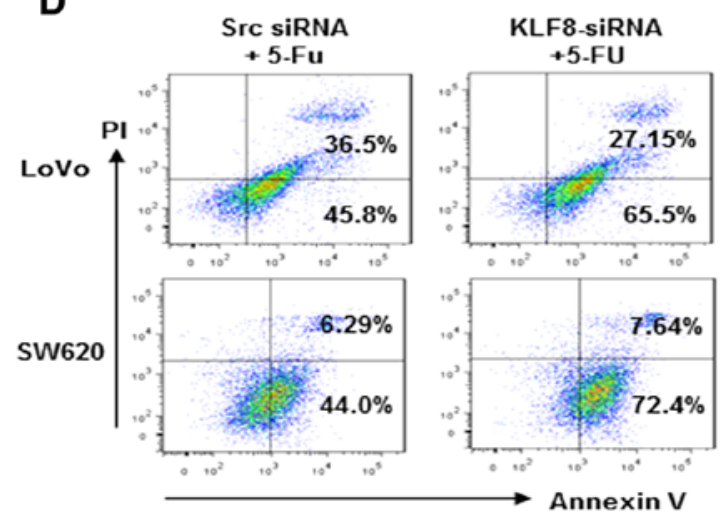

B Src siRnA
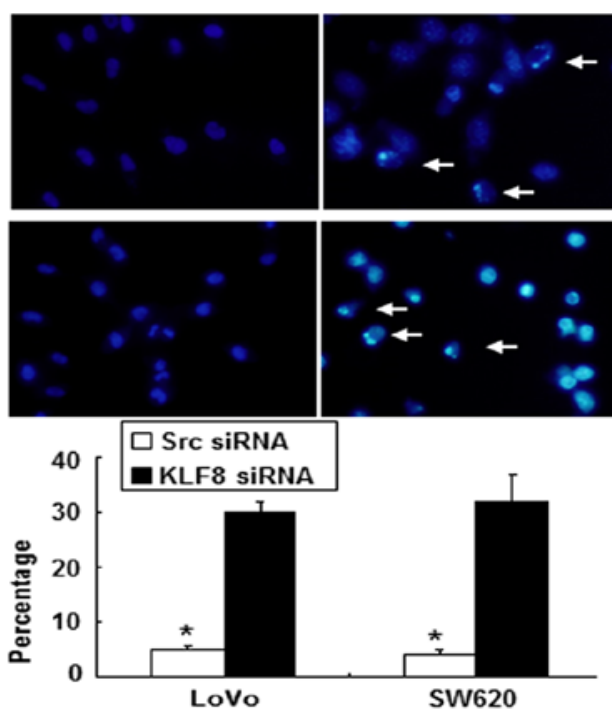

$\mathbf{E}$
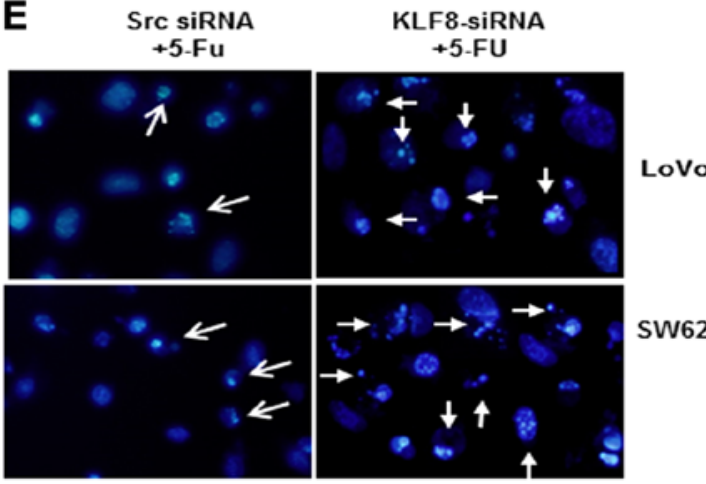

SW620

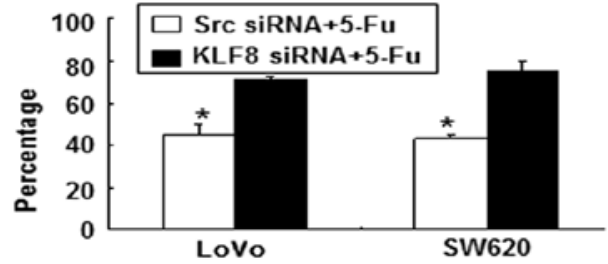

Figure 5. Repression of KLF8 induces apoptosis and sensitizes cancer cells to 5-FU. (A) Cells transfected with src siRNA or KLF8-siRNA were double-stained with Annexin V-FITC and PI, followed by flow cytometric analysis. (B) LoVo and SW620 cells were treated with src siRNA or KLF8-siRNA, the nuclei were stained with Hoechst 33258, and the cells were visualized under a fluorescent microscope (arrows show cells exhibiting nuclear fragmentation and condensed chromatin). The values are presented as the means \pm SD from three separate experiments; ${ }^{*}$ p $<0.05$. (C) The activities of caspases 3,8 and 9 were analyzed over $48 \mathrm{~h}$. The $\mathrm{OD}_{405 \mathrm{~nm}}$ was calculated individually for each group ( $\left.\mathrm{p}<0.05\right)$. (D) Apoptosis in cells treated with scr siRNA +5 -FU or KLF8-siRNA +5 -FU was determined by flow cytometric analysis. (E) Nuclei of LoVo and SW620 cells treated with src siRNA + 5-FU or KLF8-siRNA + 5-FU were stained with Hoechst 33258. The experiments were repeated three times with identical findings. 5-FU, 5-fluorouracil.

$32.58 \%$ in KLF8-siRNA-transfected LoVo cells, and similar results were found for SW620 cells (from 5.71 to $24.2 \%$ ).

Apoptotic induction was further confirmed at the individual cell level using Hoechst 33258 staining (18). We found that the ratios of cells that were positive for condensed nuclei were higher in KLF8-siRNA-transfected LoVo cells $(30 \pm 2 \%)$ compared with src siRNA-transfected LoVo cells $(5 \pm 1 \%, \mathrm{p}<0.05)$ (Fig. 5B). Similar results were observed for SW620 cells.

The activities of caspases 3,8 and 9 were then analyzed after KLF8-siRNA transfection and were normalized to the OD, which was measured at $405 \mathrm{~nm}$. As shown in Fig. 5C, the activities of caspases 3,8 and 9 were significantly increased in KLF8-siRNA-transfected cells compared with src siRNA transfected cells (Fig. 5C, p<0.05).

To assess the role of KLF8-siRNA in chemotherapyinduced apoptosis, KLF8-siRNA- or src siRNA-transfected cells were treated with or without 5 -FU [50 $\mu \mathrm{g} / \mathrm{ml}$ in normal saline (NS)] followed by flow cytometric analysis. As shown in Fig. 5D, the apoptotic index of cells treated with KLF8-siRNA + 5-FU was significantly increased relative to that of the src siRNA control cells. In addition, apoptotic morphological changes were analyzed using Hoechst 33258 staining. The ratios of LoVo cells that were positive for condensed nuclei were higher in cells treated with KLF8siRNA + 5-FU compared with the src siRNA + 5-FU control 

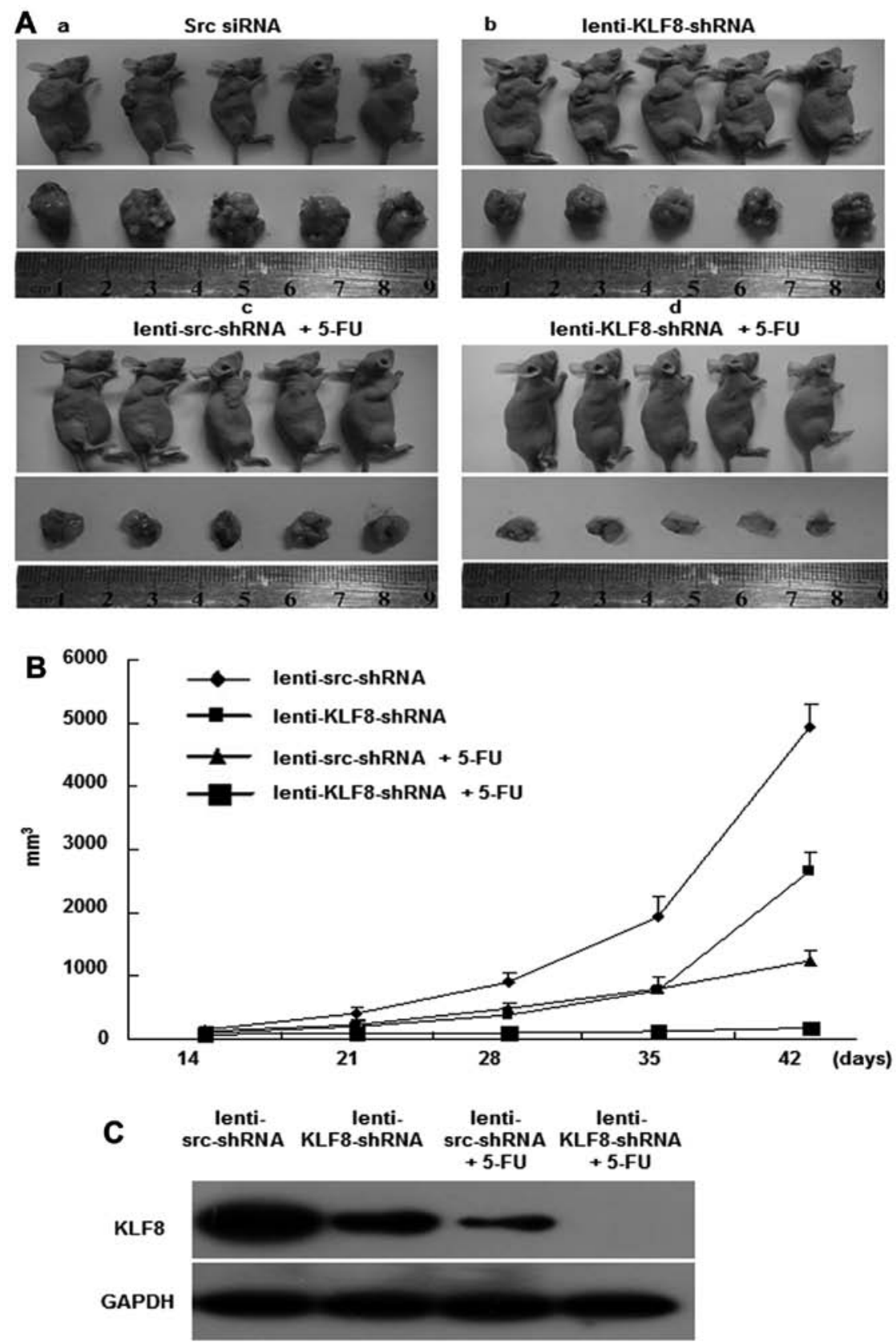

Figure 6. Suppression of KLF8 with shRNA sensitizes cancer cells to 5-FU-induced apoptosis in vivo. (A) Lentivirus-transduced LoVo cells $\left(5 \times 10^{6}\right)$ were subcutaneously injected into the right flanks of BALB/c nude mice. When the tumor nodules became visible (14 days later), $5-\mathrm{FU}$ (50 $\mu \mathrm{g} / \mathrm{kg}$, once/two days, 12 times) was given by intraperitoneal injection. The images shown were captured on day 42. (B) Tumor size was measured weekly after tumor cell inoculation in each group, and tumor growth was monitored in three dimensions and expressed as the tumor volume in cubic millimeters. The arrow indicates the time point when intraperitoneal injections were performed. The data are presented as the means \pm SD of the tumor volumes for each of the 5 animals/group. "P<0.05, lenti-KLF8-shRNA vs. lenti-src-shRNA. ${ }^{\# p}<0.05$, lenti-src-shRNA + 5-FU vs. lenti-KLF8-shRNA + 5-FU. (C) Protein extracts were prepared from pooled samples of each group, and the expression of KLF8 was detected by western blotting, with GAPDH used as the internal control. This image of western blotting was representatives of three independent experiments with identical findings. 5-FU, 5-fluorouracil.

cells $(\mathrm{p}<0.05)$ (Fig. 5E). Similar results were observed for SW620 cells.

These findings suggested that KLF8-siRNA enhanced the susceptibility of cancer cells to apoptotic triggers induced by 5-FU.

Suppression of KLF8 sensitizes cancer cells to 5-FU-induced apoptosis in vivo. To determine whether KLF8 silencing inhibits tumor development in vivo, lentivirus-transduced LoVo cells were subcutaneously injected into the right dorsal flank of nude mice that were also treated with or without 5-FU (Fig. 6A). As shown in Fig. 6B, the tumor volumes of LoVo-lentiKLF8-shRNA-injected mice were markedly smaller than that of the LoVo-lenti-src-shRNA-injected mice (a and b). Similar results were found in the 5-FU-treated mice compared with mice not treated with 5-FU (c and d). More importantly, mice injected with LoVo-lenti-KLF8-shRNA and 5-FU presented the smallest tumor nodules. In Fig. 6C, we measured KLF8 expression in the tumor tissues of four mice groups by western blotting. Obviously, KLF8 expresses the lowest in tumor 
tissues of mice injected with LoVo-lenti-KLF8-shRNA and 5-FU. Therefore, synergism between the lenti-KLF8-shRNA and 5-FU inhibited LoVo cell proliferation in vivo.

Taken together, these data indicate that targeting KLF8 with lenti-KLF8-shRNA exerted an inhibitory effect on tumorigenesis in vivo. Furthermore, inhibition of KLF8 has a synergistic effect with 5-FU treatment in the therapy of colorectal cancer.

\section{Discussion}

In the present study, we characterized the role of KLF8 in the cell growth and differentiation of colorectal cancer. We found that KLF8 is overexpressed in most colorectal cancer cell lines and cancerous tissues. Furthermore, we demonstrated the antitumor efficacy of KLF8 inhibition in cells and xenograft models, indicating that lenti-KLF8-shRNA is a novel and potent therapeutic or 5-FU-co-therapeutic agent for colorectal cancer.

KLF8 is a GT-box (CACCC)-binding, dual transcription factor that has critical roles in the regulation of cell cycle progression and in oncogenic transformation $(16,17)$. KLF8 is expressed at low levels in normal human epithelial cells but is highly overexpressed in several types of cancer cell lines that were established from human patients, including ovarian, breast, gastric and renal carcinoma cells $(16,17,27,28)$. However, to the best of our knowledge, few studies have focused on KLF8 expression in colorectal cancer. In the present study, KLF8 was expressed at higher levels in colorectal cancer than in normal tissues, and specific KLF8 knockdown in colorectal cancer cells significantly inhibited -dependent and anchorageindependent growth. These results demonstrate that KLF8 is important in CRC cell proliferation and transformation.

ERK1/2 is a member of the mitogen-activated protein kinase (MAPK) family and is known to influence cell growth, migration and invasion of various types of cancer $(2,15,29)$. However, the mechanism by which KLF8 affects ERK1/2 activation has yet to be determined. We found that U0126, a specific MEK/ERK activation inhibitor, strongly suppressed the expression of KLF8, suggesting that KLF8 is one of the major downstream effectors of ERK signaling. Furthermore, KLF8 suppression markedly inhibited constitutive ERK activation. Thus, the expression levels of pERK and KLF8 are tightly correlated during cancer progression.

F-actin has been found to reflect differentiation-related changes in cells undergoing tumorigenesis $(7,30)$. In agreement with this, we identified KLF8 as a mediator of NaB-induced pro-differentiation of colorectal cancer. KLF8 suppression increased the expression of two differentiation markers for colorectal epithelial cells, CEA and E-cadherin. Moreover, our finding that KLF8 suppression induced the maturation of F-actin filaments in cancer cells suggests the involvement of KLF8 in the dedifferentiation of cancer cells. Taken together, our data show that KLF8 suppression increases the differentiation of cancer cells.

Apoptosis is the primary means by which radiotherapy and most chemotherapy modalities kill cancer cells $(18,26)$. Our findings revealed that KLF8-siRNA directly induced apoptosis in vitro and that the combination of lenti-KLF8-shRNA and 5-FU treatment provided a synergistic antitumor growth effect in vivo. Although a few studies have shown the antitumor effect of KLF8 inhibition in different types of cancer, to the best of our knowledge, no reports have shown the therapeutic value of the lenti-KLF8-shRNA in colorectal cancer.

In conclusion, the present study shows that higher levels of KLF8 are expressed in colorectal cancer tissues compared with normal tissues. In addition, KLF8 suppression induces cell differentiation, inhibits cell growth and promotes apoptosis in colorectal cancer cells in vitro. Furthermore, lenti-KLF8-shRNA hinders tumor progression and outright regression when combined with 5-FU in vivo. Thus, targeting of KLF8 by RNA interference may have a promising role in the management of colorectal cancer.

\section{Acknowledgements}

The present study was supported by grants from the National Natural Science Funds of China (nos. 81172057 and 81272761), and the 'President Foundation of Nanfang Hospital, Southern Medical University' (nos. 2012B009 and 2013Z007) and by high-level, topic-matching funds of Nanfang Hospital (nos. 201347 and G201227).

\section{References}

1. Brenner H, Kloor M and Pox CP: Colorectal cancer. Lancet 383: 1490-1502, 2014

2. Yu LF, Wang J, Zou B, Lin MC, Wu YL, Xia HH, Sun YW, Gu Q, He H, Lam SK, et al: XAF1 mediates apoptosis through an extracellular signal-regulated kinase pathway in colon cancer. Cancer 109: 1996-2003, 2007.

3. Kopelovich L: Heritable colorectal cancer and cancer genes: Systemic expressions. Mol Carcinog 8: 3-6, 1993.

4. Ponce NA, Tsui J, Knight SJ, Afable-Munsuz A, Ladabaum U, Hiatt RA and Haas JS: Disparities in cancer screening in individuals with a family history of breast or colorectal cancer. Cancer 118: 1656-1663, 2012.

5. Doubeni CA, Major JM, Laiyemo AO, Schootman M,Zauber AG, Hollenbeck AR, Sinha R and Allison J: Contribution of behavioral risk factors and obesity to socioeconomic differences in colorectal cancer incidence. J Natl Cancer Inst 104: 1353-1362, 2012.

6. Fernandez E, La Vecchia C, Talamini R and Negri E: Joint effects of family history and adult life dietary risk factors on colorectal cancer risk. Epidemiology 13: 360-363, 2002.

7. Wang J, Yang Y, Xia HH, Gu Q, Lin MC, Jiang B, Peng Y, Li G, An X, Zhang Y, et al: Suppression of FHL2 expression induces cell differentiation and inhibits gastric and colon carcinogenesis. Gastroenterology 132: 1066-1076, 2007.

8. Zhang W, Jiang B, Guo Z, Sardet C, Zou B, Lam CS, Li J, He M, Lan HY, Pang R, et al: Four-and-a-half LIM protein 2 promotes invasive potential and epithelial-mesenchymal transition in colon cancer. Carcinogenesis 31: 1220-1229, 2010.

9. Warren RS, Yuan H, Matli MR, Gillett NA and Ferrara N: Regulation by vascular endothelial growth factor of human colon cancer tumorigenesis in a mouse model of experimental liver metastasis. J Clin Invest 95: 1789-1797, 1995.

10. Furue H, Matsuo K, Kumimoto H, Hiraki A, Suzuki T, Yatabe Y, Komori K, Kanemitsu Y, Hirai T, Kato T, et al: Decreased risk of colorectal cancer with the high natural killer cell activity $N K G 2 D$ genotype in Japanese. Carcinogenesis 29: 316-320, 2008.

11. Guo Z, Zhang W, Xia G, Niu L, Zhang Y, Wang X, Zhang Y, Jiang B and Wang J: Sp1 upregulates the four and half lim 2 (FHL2) expression in gastrointestinal cancers through transcription regulation. Mol Carcinog 49: 826-836, 2010.

12. Lee PL, Gelbart T, West C, Adams M, Blackstone R and Beutler $\mathrm{E}$ : Three genes encoding zinc finger proteins on human chromosome 6p21.3: Members of a new subclass of the Krüppel gene family containing the conserved SCAN box domain. Genomics 43: 191-201, 1997. 
13. Le Magnen C, Bubendorf L, Ruiz C, Zlobec I, Bachmann A, Heberer M, Spagnoli GC, Wyler S and Mengus C: Klf4 transcription factor is expressed in the cytoplasm of prostate cancer cells. Eur J Cancer 49: 955-963, 2013.

14. Yang Y, Nakagawa H, Tetreault MP, Billig J, Victor N, Goyal A, Sepulveda AR and Katz JP: Loss of transcription factor KLF5 in the context of p53 ablation drives invasive progression of human squamous cell cancer. Cancer Res 71: 6475-6484, 2011.

15. Ellenrieder V, Buck A, Harth A, Jungert K, Buchholz M, Adler G, Urrutia R and Gress TM: KLF11 mediates a critical mechanism in TGF-beta signaling that is inactivated by Erk-MAPK in pancreatic cancer cells. Gastroenterology 127: 607-620, 2004.

16. Wang X, Zheng M, Liu G, Xia W, McKeown-Longo PJ, Hung MC and Zhao J: Krüppel-like factor 8 induces epithelial to mesenchymal transition and epithelial cell invasion. Cancer Res 67: 7184-7193, 2007.

17. Fu WJ, Li JC, Wu XY, Yang ZB, Mo ZN, Huang JW, Xia GW, Ding Q, Liu KD and Zhu HG: Small interference RNA targeting Krüppel-like factor 8 inhibits the renal carcinoma 786-0 cells growth in vitro and in vivo. J Cancer Res Clin Oncol 136 $1255-1265,2010$.

18. Wu Y, Guo Z, Zhang D, Zhang W, Yan Q, Shi X, Zhang M, Zhao Y,Zhang Y, Jiang B, et al: A novel colon cancer gene therapy using rAAV mediated expression of human shRNA-FHL2. Int J Oncol 43: 1618-1626, 2013.

19. Li JC, Yang XR, Sun HX, Xu Y, Zhou J, Qiu SJ, Ke AW, Cui YH, Wang ZJ, Wang WM, et al: Up-regulation of Krüppel-like factor 8 promotes tumor invasion and indicates poor prognosis for hepatocellular carcinoma. Gastroenterology 139: 2146-2157.e12, 2010.

20. Wang TB, Hu BG, Liu DW, Shi HP and Dong WG: The influence of lentivirus-mediated CXCR4 RNA interference on hepatic metastasis of colorectal cancer. Int J Oncol 44: 1861-1869, 2014.

21. Dent P: Multi-kinase modulation for colon cancer therapy. Cancer Biol Ther 14: 877-878, 2013.
22. Orchel A, Dzierzewicz Z, Parfiniewicz B, Weglarz L and Wilczok T: Butyrate-induced differentiation of colon cancer cells is PKC and JNK dependent. Dig Dis Sci 50: 490-498, 2005.

23. Bajenova O, Chaika N, Tolkunova E, Davydov-Sinitsyn A, Gapon S, Thomas P and O'Brien S: Carcinoembryonic antigen promotes colorectal cancer progression by targeting adherens junction complexes. Exp Cell Res 324: 115-123, 2014.

24. Yan $\mathrm{H}$, Wang X, Niu J, Wang Y, Wang P and Liu Q: Anti-cancer effect and the underlying mechanisms of gypenosides on human colorectal cancer SW-480 cells. PLoS One 9: e95609, 2014.

25. Yu J, Qiao L, Zimmermann L, Ebert MP, Zhang H, Lin W, Röcken C, Malfertheiner P and Farrell GC: Troglitazone inhibits tumor growth in hepatocellular carcinoma in vitro and in vivo. Hepatology 43: 134-143, 2006.

26. Tu SP, Liston P, Cui JT, Lin MC, Jiang XH, Yang Y, Gu Q Jiang SH, Lum CT, Kung HF, et al: Restoration of XAF1 expression induces apoptosis and inhibits tumor growth in gastric cancer. Int J Cancer 125: 688-697, 2009.

27. Schnell O, Romagna A, Jaehnert I, Albrecht V, Eigenbrod S, Juerchott K, Kretzschmar H, Tonn JC and Schichor C: Krüppel-like factor 8 (KLF8) is expressed in gliomas of different WHO grades and is essential for tumor cell proliferation. PLoS One 7: e30429, 2012

28. Lu H, Wang X, Urvalek AM, Li T, Xie H, Yu L and Zhao J Transformation of human ovarian surface epithelial cells by Krüppel-like factor 8. Oncogene 33: 10-18, 2014.

29. Bromberg KD, Kluger HM, Delaunay A, Abbas S, DiVito KA, Krajewski S and Ronai Z: Increased expression of the E3 ubiquitin ligase RNF5 is associated with decreased survival in breast cancer. Cancer Res 67: 8172-8179, 2007.

30. Franovic A, Holterman CE, Payette J and Lee S: Human cancers converge at the HIF-2alpha oncogenic axis. Proc Natl Acad Sci USA 106: 21306-21311, 2009. 